\title{
Miller Fisher Syndrome - A Case Report
}

\author{
Miguel Pires, Joana Monteiro, Sonia Chan, Luisa Pinto \\ Centro Hospitalar de Leiria
}

\section{Introduction}

Miller Fisher Syndrome (MFS) is a rare variant of GBS (Guillain-Barre Syndrome). It involves both adults and children and its mostly a clinical diagnosis. It comprises a classic triad of ophthalmoplegia, ataxia and areflexia which all develop over a period of few days. This triad was first described by James Collier in 1932 and it was subsequently reported as a variant of GBS by Charles Miller Fisher in three clinical cases in $1956^{1}$.

MFS is a geographically variable variant of GBS observed in about $1 \%$ to $5 \%$ of all GBS cases in Western countries, yet up to $19 \%$ and $25 \%$ in Taiwan and Japan respectively ${ }^{2}$. There is a male predominance at a ratio of 2:1 and a mean age of 43.6 years, although cases of MFS have been reported in all age groups ${ }^{2}$.

Unlike the classic ascending weakness/paralysis that is characteristic of GBS, neurological deficits follow a top down pattern in MFS, starting with diplopia in the eyes; caused by external ophthalmoplegia - the most common presenting symptoms ${ }^{2,4}$. In a clinical series of 50 consecutive cases of MFS in Japan it was discovered that $78 \%$ of cases presented initially with diplopia, $46 \%$ with ataxia, and $34 \%$ with both. Other abnormalities reported, albeit less frequently, were limb dysesthesia; blepharoptosis; face, bulbar, and pupillary palsies; mild (grade 4) motor weakness; and micturition disturbance ${ }^{2}$.
As in GBS an antecedent infectious illness can be identified. Upper respiratory infection is the most commonly described prodromal entity followed by gastrointestinal illness ${ }^{3}$. Campylobacter jejuniand Haemophilus influenza have been the most commonly implicated pathogens. Other pathogens, including Mycoplasma pneumonia and Cytomegalovirus, have been associated too.

An acute onset is typical of MFS, beginning with neurologic symptoms approximately 8-10 days (range of 1-30) following the antecedent illness ${ }^{2,5}$. The disease then progresses until a clinical nadir is reached approximately 6 days (range of 2-21) after the initial neurologic symptoms ${ }^{2}$. The recovery period is marked by gradual improvement and often resolution of symptoms; although rarely, serious complications such as respiratory failure or cardiac arrhythmia (that are common in GBS, with $30 \%$ of cases requiring ventilator support) have been reported ${ }^{5}$.

The following case presents key clinical features of MFS. Familiarity with this rare syndrome will clue the clinician to consider MFS in patients presenting with areflexia, ataxia, and ophthalmic symptom.

Keywords: Areflexia. Ataxia. Guillain-Barré syndrome. Miller Fisher. Ophthalmoplegia.

Palabras clave: Arreflexia. Ataxia. Síndrome de Guillain-Barré. Miller Fisher. Oftalmoplejía.

\section{Case report}

Male, 55-year-old, presented to the emergency department (ED) with headache, difficulty in vision and unsteadiness in walking for 5 days. There were no complains of unconsciousness, convulsion, speech problems, hearing defects, vertigo, tinnitus, dysphagia or hoarseness of voice. There were no complains of motor weakness elsewhere in the body, sensory symptoms or bowel or bladder symptom.

He had a history of bipolar disease and alcoholism, but he was not taking any medication.

Upon physical examination hypertension was present (174/130 $\mathrm{mmHg}$ ). Other vital signs were in the normal range. The patient had normal higher function. Neurological examination revealed marked ophthalmoplegia with severe decreased movements on lateral, medial and upward gaze. The pupils were bilaterally equal, semi-dilated and not reacting to light or accommodation. There was no ptosis. Decreased visual acuity in both eyes (Right eye 20/50 and Left eye 20/10 on Snellen's chart). The visual field and fundus both were normal. Rest of the cranial nerves were normal to examination. A global areflexia was present, and muscle strengths testing was normal. Sensory examination was normal. The finger nose test and the knee heel test were normal. Dysdiadochokinesia was absent. Romberg's sign was negative. The gait was ataxic and broad based but possible with a cane.

Patient was admitted for further investigation and treatment.
On the second day of hospital admission the patient developed bilateral ptosis and eye movements were restricted in all direction. No change in muscle strength but patient was unable to walk.

Laboratory testing revealed normal complete blood count, comprehensive metabolic panel, thyroid stimulating hormone, and cardiac markers. Urine toxicology screens were negative for common substances of abuse. There was no elevation in erythrocyte sedimentation rate, C-reactive protein, or creatine kinase; HIV and RPR serologies were negative. On note, laboratory investigations for some of the more commonly implicated pathogens in MFS (Campylobacter jejuni, Haemophilus influenza, Mycoplasma pneumonia, or cytomegalovirus) were not undertaken. A lumbar puncture was performed and revealed cytoalbuminologic dissociation in the CSF with normal cell counts and an elevated protein count of $131.0 \mathrm{mg} / \mathrm{dL}$. Nerve Conduction Velocity (NCV) study showed mixed demyelinating (both motor and sensory) polyneuropathy, with an $\mathrm{H}$-reflex detected. Contrast enhanced brain magnetic resonance imaging did not reveal areas of contrast enhancement or parenchymal abnormalities. Based on clinical presentation of ataxia and areflexia, and history of alcohol abuse, there was a concern for Wernicke's encephalopathy and the patient was started on intravenous thiamine $(500 \mathrm{mg}$ every 8 hours). A rapid response was expected; however, this was not observed. The other two main competing diagnoses were an atypical presentation of GBS or MFS. Tests were ordered to look for antibo- 
dies that would support or refute each of these diagnoses. Meanwhile, decisions to start further treatment were made because the patient didn't improve. The intravenous thiamine was discontinued, and the patient was treated with intravenous immune globulin (IVIG) for seven days to treat for possible MFS. Serum antibody test results were positive for GQ1b IgG and the diagnosis of MFS was confirmed. The nadir of the patient's symptoms was reached by day seven. Physical, occupational, and speech therapy specialists were working daily with the patient and he began to exhibit steady improvement. He was able to walk without assistance by the tenth hospital day and on the twelfth day he was discharged.

\section{Discussion}

MFS is known for the characteristic triad of opthalmoplegia, ataxia, and areflexia. It is considered a variant of GBS, which is also known as acute idiopathic neuritis. An increasing body of evidence suggests that a rather wide range of neurological features may be present and significant overlap exists with other forms of GBS.

Berlit et al reviewed 223 cases of MFS: the first symptom was diplopia (38.6\%) or ataxia (20.6\%). Areflexia was present in $81.6 \%$ of cases. The cranial nerves other than III were involved in 127 cases (56.9\%): cranial nerves VII (45.7\%), IX and $X(39.9 \%)$, and XII (13\%) were involved. In 53 cases, tetraparesis occurred. Elevated CSF protein was present in 134 patients (64.4\%). CSF findings were normal in 56 patients, and 18 patients had mild pleocytosis. The prognosis of MFS was good. Recovery occurred after a mean time of 10.1 weeks. Residual symptoms were present in 74 cases (33.2\%), recurrence of MFS was reported in 7 patients, and 8 patients died ${ }^{6}$. Fross et al reported 10 patients with typical MFS electrophysiological abnormalities characteristic of axonal neuropathy or neuronopathy with predominant sensory nerve changes in the limbs and motor damage in the cranial nerves?

The differential diagnosis of MFS includes Wernicke's encephalopathy and brainstem encephalitis, but these are associated with altered mental status. In addition, patients with Wernicke's encephalopathy usually have nystagmus, a feature not associated with MFS. Other entities to consider in the differential diagnosis of MFS are myasthenia gravis and other neuromuscular junction disorders.

Appropriate laboratory and clinical electrophysiological testing with repetitive nerve stimulation helps to differentiate MFS from a neuromuscular junction disorder ${ }^{8}$. The combination of absent or reduced sensory responses on clinical electrophysiology testing and elevated protein with a normal white blood cell in CSF could lead to the diagnosis of MFS ${ }^{9}$. The diagnosis could be confirmed by positive anti-GQ1b antibody testing with a high level of sensitivity (well over $90 \%$ of patients with MFS have this antibody) and specificity. However, radiological imaging were unremarkable in most cases of MFS, lesions in the brainstem and spinocerebellar tracts had been reported in few patients ${ }^{10}$.

MFS is generally regarded as a self-limiting benign condition. All of 28 untreated MFS patients in the largest published case series returned to normal activities 6 months after the neurological onset. The respective median (range) periods between neurological onset and the disappearance of ataxia and ophthalmoplegia were reported as 32 (8-271) and 88 (29-165) days. However, cases progressing to respiratory failure and requiring mechanical ventilation have also been described, particularly in children. Other serious complications reported include coma, ballism, cardiomyopathy from dysautonomia, lactic acidosis, and pain ${ }^{5}$.

In conclusion, MFS is a clinical diagnosis with a classic triad (ataxia, areflexia and opthalmoplegia) and the early recognition improves the outcome of this highly limiting patients'condition.

\section{Bibliography}

1. Fisher M. An Unusual Variant of Acute Idiopathic Polyneuritis (Syndrome of Ophthalmoplegia, Ataxia and Areflexia). N Engl J Med [Internet]. 1956 Jul 12 [cited 2017 Dec 6];255(2):57-65. Available from: http://www.nejm.org/doi/abs/10.1056/ NEJM195607122550201.

2. Mori M, Kuwabara S, Fukutake T, Yuki N, Hattori T. Clinical features and prognosis of Miller Fisher syndrome. Neurology [Internet]. 2001 Apr 24 [cited 2017 Dec 6];56(8):1104-6. Available from: http://www.ncbi.nlm.nih.gov/pubmed/11320188.

3. Yepishin IV, Allison RZ, Kaminskas DA, Zagorski NM, Liow KK. Miller Fisher Syndrome: A Case Report Highlighting Heterogeneity of Clinical Features and Focused Differential Diagnosis. Hawaii J Med Public Health [Internet]. 2016 Jul [cited 2017 Nov 30];75(7):196-9. Available from: http://www.ncbi.nlm.nih.gov/pubmed/27437164.

4. Snyder LA, Rismondo V, Miller NR. The Fisher Variant of Guillain-Barré Syndrome (Fisher Syndrome). J Neuro-Ophthalmology [Internet]. 2009 Dec [cited 2017 Dec 6];29(4):312-24. Available from: http://www.ncbi.nlm.nih.gov/pubmed/19952907.

5. Lo YL. Clinical and immunological spectrum of the Miller Fisher syndrome. Muscle Nerve [Internet]. 2007 Nov;36(5):615-27. Available from: http://www.ncbi.nlm.nih. gov/pubmed/17657801.

6. Berlit P, Rakicky J. The Miller Fisher syndrome. Review of the literature. J Clin Neuroophthalmol [Internet]. 1992 Mar;12(1):57-63. Available from: http://www.ncbi. nlm.nih.gov/pubmed/1532603.

7. Fross RD, Daube JR. Neuropathy in the Miller Fisher syndrome: Clinical and electrophysiologic findings. Neurology [Internet]. 1987 Sep 1;37(9):1493-1493. Available from: http://www.ncbi.nlm.nih.gov/pubmed/2819783.

8. Kuwabara S, Asahina M, Nakajima M, Mori M, Fukutake T, Hattori T, et al. Special sensory ataxia in Miller Fisher syndrome detected by postural body sway analysis. Ann Neurol. 1999;45(4):533-6.

9. Sever M, Aksay E, Gulec F. The diagnosis is made only with suspicion: Miller fisher syndrome. Hong Kong J Emerg Med. 2011;18(6):428-31.

10. Partha Pratim Das1, Sarmistha Biswash1, Md. Enamul Karim2, Nigar Sultana Ahmed3, Badrul Islam4 S, Kumar Mandal5 AHMRS. Miller Fisher Variant of GuillainBarré Syndrome: A Case Report \& Clinical Reviewtle. BSMMU J 2012 [Internet]. 2012 Jun 29 [cited 2017 Nov 30];5(69-71). Available from: http://www.banglajol. info/index.php/BSMMUJ/article/view/11028. 